\title{
Spontaneous retroperitoneal hematoma associated with anticoagulation therapy and antiplatet therapy: Two centers experiences
}

\author{
Abdulmuttalip Simsek ${ }^{1}$, Faruk Ozgor ${ }^{1}$, Bahar Yuksel ${ }^{2}$, Ercan Bastu ${ }^{2}$, Mehmet Fatih Akbulut ${ }^{1}$, \\ Onur Kucuktopcu ${ }^{1}$, Omer Sarilar ${ }^{1}$, Ahmet Yalcin Berberoglu ${ }^{1}$, Zafer Gokhan Gurbuz ${ }^{1}$ \\ ${ }^{1}$ Haseki Research and Training Hospital, Department of Urology; \\ 2 Istanbul University, School of Medicine, Department of Obstetrics and Gynecology.
}

\begin{abstract}
Summary Background: To analyze the characteristics of the patients with diagnosis of spontaneous retroperitoneal hematoma associated with anticoagulation therapy and antiplatet therapy. Methods: From January 2006 to March 2013, 9 patients (6 from Haseki Training and Research Hospital - Urology Department and 3 from Istanbul Medical Faculty Gynecology and Obstetric Department) were included in the study. Patients charts including sex, age, comorbidities, main complaint, and medication intake were examined. Also initial hemoglobin level, initial International Normalized Ratio level, red blood cells and fresh frozen plasma units transfused were evaluated.

Results: Median age was 60 year-old. Abdominal pain and flank pain were common symptoms. Eight patients were taking only anticoagulation therapy, 2 only antiplatet therapy and 1 both anticoagulation and antiplatet therapy. Median initial hemoglobin value was $9,0 \mathrm{~g} / \mathrm{dL}$ and median International Normalized Ratio level was 3.2 Patients were evaluated by abdominal ultrasonography or abdominal computer tomography. Seven patients were treated conservatively. Only one patient died because of septic shock with a mortality ratio of $11 \%$.

Conclusion: Despite benefits of anticoagulation and antiplatet theraphy these agents have serious side-affects as retroperitoneal hemorrhage in elderly patients taking multi-drug medication.
\end{abstract}

KEY WORDS: Spontaneous retroperitoneal hematoma; Anticoagulation therapy; Antiplatet therapy.

Submitted 14 January 2014; Accepted 30 June 2014

\section{INTRODUCTION}

Spontaneous retroperitoneal hematoma (SRH) is a rare but serious clinical condition described as bleeding into the retroperitoneal area without associated trauma or surgical manipulation (1). The most common causes of SRH are renal tumors, vascular diseases and anticoagulation treatments (2). Despite the benefits of anticoagulation theraphy
(ACT) and antiplatet theraphy (APT) were well described in stroke, deep vein thrombosis and pulmonary embolism, these agent may be cause of bleeding anywhere in the human body (3). Once when retroperitoneal haemorrhage occurs, the patient's condition abruptly deteriorates requiring assistance in emergency due to hypotension, weakness and flank pain. Also hematoma induce symptoms due to the compression of adjacent structures. In this paper we aim to present the exprience of two center (Haseki Training And Research Hospital - Urology Department and Istanbul Medical Faculty - Gynecology and Obstetric Department) about SRH associated with ACT and APT.

\section{Materials AND Methods}

In two academic tertiary care center with high volume emergency department, patients charts were evaluated retrospectively. From January 1, 2006 to March 31, 2013; 6 patients in Haseki Training And Research Hospital - Urology Department (HSKU) and 3 patients in Istanbul Medical Faculty - Gynecology and Obstetric Department (IMFGO) with diagnosis of SRH associated with ACT and APT were included study. Sex and age of patients, comorbidities, main complaint and medication intake were analyzed. Also history of trauma and surgical manipulation was investigated carefully. Patients with history of surgical manipulation up to three months prior to the time of application were excluded from the study. Initial hemoglobin level, initial International Normalized Ratio (INR) level, red blood cells (RBC) and fresh frozen plasma (FFP) units transfused were evaluated. Additionally diagnostic imaging methods were recorded. All patients charts were rewieved by two authors for variables defined before data collection. Microsoft Excel 2010 software (Microsoft Corporation, Redmond, WA) was used for data entry.

\section{RESULTS}

Nine patients were included in the study (Table 1). Four were males and five were females with median age 60 years (range from 37 to 77 years). Abdominal and flank 
Table 1.

Characteristics of mine patients.

\begin{tabular}{|c|c|c|c|c|c|c|c|c|c|c}
\hline & Age & Gender & Symptoms & $\begin{array}{c}\text { Drug } \\
\text { type }\end{array}$ & $\begin{array}{c}\text { Initial } \\
\text { hgb }\end{array}$ & $\begin{array}{c}\text { INR } \\
\text { level }\end{array}$ & $\begin{array}{c}\text { RBC } \\
\text { Transfusion } \\
\text { (number } \\
\text { of pocket) }\end{array}$ & $\begin{array}{c}\text { FFP } \\
\text { Transfusion } \\
\text { (number } \\
\text { of pocket) }\end{array}$ & Surgery & Co-morbidities \\
\hline P-1* & 64 & F & Pain, Weakness & ACT & 9.8 & 3,2 & 2 & + & - & HT, DM \\
\hline P-2* & 53 & F & Pain, Weakness & ACT+ APT & 12.1 & 5 & - & + & - & CVE, HT \\
\hline P-3* & 71 & F & Pain,Weakness, Dizziness & ACT & 7.4 & 3,6 & 2 & + & - & P.ULCUS, HT \\
\hline P-4 & 60 & F & Pain & ACT & 8.2 & 1,2 & 2 & + & - & DM,CVE \\
\hline P-5 & 48 & M & Pain, Weakness, Dyspnea & ACT & 5.9 & 2 & 9 & + & - & HT, CKD \\
\hline P-6 & 43 & M & Pain, Abdominal Maas, Flank Coloration & ACT & 7.6 & 3,5 & 3 & + & - & - \\
\hline P-7 & 77 & M & Pain, Weakness & APT & 11.4 & 1,4 & - & - & - & DVT \\
\hline P-8 & 64 & M & Pain,Weakness, Dizziness & APT & 10.1 & 1,3 & 4 & + & + & HT, CKD \\
\hline P-9 & 37 & F & Pain, Dyspepsia & ACT & 9.0 & 5,8 & 6 & + & + & LE, HT \\
\hline
\end{tabular}

*: Patients treated in Istanbul Medical Faculty - Gynecology and Obstetric Department; RBC: Red Blood Cell; FFP: Fresh Frozen Plasma; ACT: Anticoagulation Theraphy;

APT: Antiplatet Theraphy; HT: Hypertension; DM: Diabetes mellitus; CVE: Cerebrovasculer Event; LE: Lupus Erythematosus; CKD: Chronic Kidney Disease; DVT: Deep Vein Thrombosis.

pain, weakness, dizziness were the most common symptoms. Also acute dyspnea and dyspepsia were described. Abdominal mass and flank discoloration was seen only in one patient. One patient (11\%) was taking a combination of ACT and APT (acetylsalicylic acid plus warfarin). Six patients were taking only ACT includig warfarin or heparin. Two patients were taking only APT (acetylsalicylic acid). Almost all patients (88\%) were receiving multiple medication simultaneously. Initial mean hemoglobin value was $9.05 \mathrm{~g} / \mathrm{dL}$ and ranged 5.9-12.1 g/dL. Median International Normalized Ratio (INR) level was 3.2 (range 1.2-5.8) with supratherapeutic level (>3) in 5 patients.

The diagnosis was based on imaging method but two patients (22\%) were initially misdiagnosed. The first diagnosis of these two patient was endometrioma and pelvic mass respectively, depending on abdominal ultrasonography. Abdominal ultrasonography was performed in all patients and contrast-enhanced abdominal CT was performed in 7 of 9 patients. Due to the high creatinine level two patients were evaluated by non-contrast enhanced computer tomography. Angiography was performed in 3 patients but no pathological finding was described. Magnetic Resonance Imaging was not used.

Seven patients were treated conservatively. Six patients were transfused with red blood cells, ranging from 2 to 9 units, to elevate hemoglobin level. Median number of packed red blood cells transfused was 2.7 unit per patient. Two patients did not require blood transfusion. Vitamin K and FFP was used to correct coagulopathy in 7 patients. FFP was used in one patients to avoid side effects of RBC transfusion. One patient underwent surgery in emergency deparment because of continuous decrease of hemoglobin level. The patient had chronic renal failure with non-functional hydronephrotic kidney and underwent dialysis programme. Nephrectomy and clot evacuation was performed (Figure 1). Perioperative and post operative period were uneventful. One patient underwent elective surgery after hemodynamic stabilisation. Despite the normal hemoglobin level, patient fever was elevated $>38^{\circ} \mathrm{C}$ a week after the hospitalisation even on broad-spectrum
Figure 1.

Massive retroperitoneal hematoma:

patient underwent unsuccesful clot evacuation.

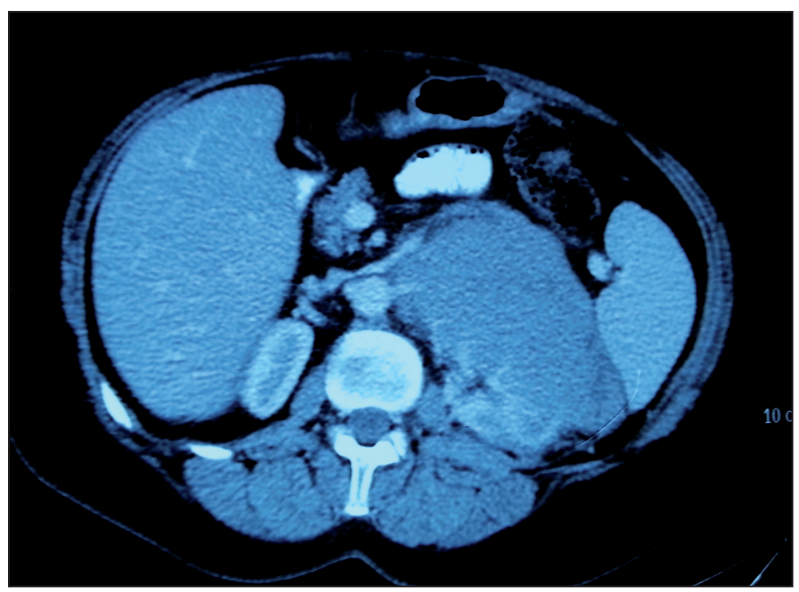

Figure 2.

Retroperitoneal hematoma in a patient who underwent a dialysis programme because of cystic disease.

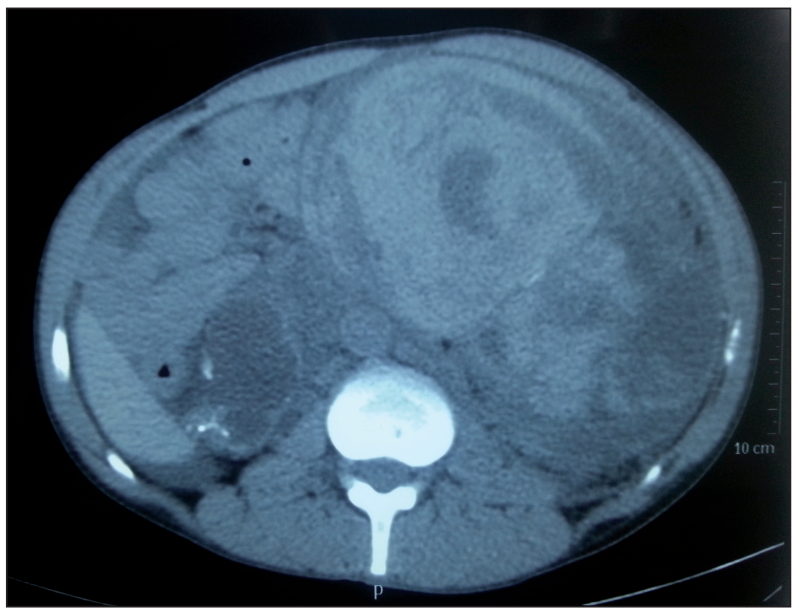


antibiotics. After achievement of normal INR level, clot evacuation, abscess drainage and, if necessary, nephrectomy was planned. Clots covered all the retroperitoneal area including aorta and superior mesenteric artery. Despite consultation of the general surgeon clot evaculation failed (Figure 2) and on the sixth day after surgery septic shock developed and the patient died.

After achievement of stable hemoglobin and coagulation levels, patients were discharged. Mean hospitalisation was 5.8 day ranging from 3 to 18 days. Follow up with imaging modalities was performed in 8 patients exluding the patient who underwent nephrectomy. Size of hematoma was decreased in 6 patients and stable in 2 patients at the discharge.

\section{Discussion}

Spontaneous retroperitoneal hematoma (SRH) is a well defined lethal entity but the pathogenetic mechanism is not clearly understood due to the rarity of the disease (4). The underlying pathology of SRH could be ignored by physicians because of its fatality and the requirement of emergency intervention. Certainly rupture of organs or vessels causes bleeding in to the retroperitoneal space without trauma or surgical manupilation. The definitive pathology is still unkown but several hypotheses have been proposed including small vessel arteriosclerosis, anticoagulation induced immune microangiopathy and forceful vascular strain (5).

Dougal and collegues investigated etiology of SRH and renal tumors including renal cell cancer and angiomyolipomas were identified as the reason of $58 \%$ of all cases. Vascular pathologies and infectious diseases played second and third role with $18 \%$ and $10 \%$ respectively (6). These findings were supported by Zhang et al. who review the english literature from 1985 to 1999 (7). Differently Sunga reported a study about SRH including 89 cases and $64 \%$ of patients were on anticoagulation therapy (8).

Anticoagulation treatment and antiplatet treatment has a essantial role for prevention of thrombosis and thromboembolism in acute myocardial infarctus, deep vein thrombosis and pulmonary embolism. Warfarin is the most widely preferred anticoagulation agent that interfere on blood caogulation by inhibiting vitamin K epoxide reductase.

During the initial stage of treatment, checking of INR may be required daily and frequent testing can be prolonged until the patient has stable therapeutic INR levels on an unchanged warfarin dose. By the fact warfarin interact with many commonly used medications and foods particularly vegetables. Also kidney failure or liver failure modify the effect of warfarin (9). Acetylsalicylic acid inhibit on cyclooxigenase and have antipyretic, anti inflammatory and analgesic affect. Most common side affects are seen in gastrointestinal tract but prolonged bleeding time and thrombocytopenia are other side affect especially in high dose use (10). Drug interaction is common for both drugs and we believe that is critical for hemorragic events. In our study 10 of our patients received multidrug treatment for hypertension, diabetes mellitus, cerebrovasculer event, lupus erythematosus and gastric ulcer. On the other hand our population mean age was $57.44 \pm 13.20$ years and five patients were older than 60. Senile systemic changes affect on drug metabolism. Reduced intestinal passage, atrophy of intestinal villi, chronic liver disease or chronic kidney disease alter the metabolism of drugs. Also systemic diseases as hypertension, diabetes mellitus, and hyperlipidemia disrupt the structure of the vascular wall and facilitate hemorrhage.

Diagnosis of SRH mostly depends on presentation symptoms and radiological studies (11). Although Lenk triad including acute flank pain, tenderness and symptoms of internal bleeding was described for SRH, symptoms may show a wide range from hip-leg pain to cardiovascular collapse. Generalized weakness, headache, dyspnea, syncope and altered mental status are less common symptoms associated with the degree and the duration of bleeding.

Particularly in patients with stable cardiovascular status, the mass of the hematoma develops a pressure on adjacent structures such as small intestine or stomach causing constipation and dyspepsia (12).

Abdominal computer tomography and ultrasonography are the most common radiological modalities for define SRH (13).

Although its non invasive, rapid and reproducible nature, ultrasonography is operator dependent and has limited capacity to define hematoma and its relation with adjacent organs. Diagnostic accuracy of CT scan is higher and give more valuable information about size, relation with adjacent organs and also underlying pathology of hematoma. Magnetic resonance imaging is helpful to differentiate blood from tumor but is performed only for patients in stable condition (14).

Managament of SRH is mostly depending on degree of bleeding and underlying pathology. If the patient's condition was deteriated or underlying pathology was renal tumor, nephrectomy was suggested (15), but clinical condition is much more complicate if SRH is associated with ACT or APT. Decision is challenging for physician because on one side there is a patient with bleeding requiring surgical intervention, on the other side surgical intervention can contribute to further bleeding.

Conservative treatment is also supported in patients with a stable condition. Bosniak et al. demonstrated that operative exploration is not essential for all unexplained cases (16). Also hematoma evacuation is performed only for patients if hematoma become focus of infection or with significant compressive symptoms.

\section{Conclusion}

In this paper we report experience about SRH associated with ACT and APT in two high volume tertiary centers. Particularly in elderly patients taking multidrug medication, side effects of ACT and APT are more frequent. Physicians must be aware about bleeding complications and have a good knowledge of its clinical presentations and treatment. If the patient's condition is stable in retroperitoneal hemorrhage, conservative treatment is a feasible method to protect patient from unnecessary surgical manipulation. 


\section{References}

1. Melde SL. Enoxaparin induced retroperitoneal hematoma. Ann Pharmacother. 2003; 37:822- 824.

2. Daskalopoulos G, Karyotis I, Heretis I, et al. Spontaneous perirenal hamorrhage:A 10-year exprience at our institution. Int Urol and Nephr. 2004; 36:15-9.

3. Moudouni SM, En-Nia I, Patard JJ, et al. Spontaneous subcapsular renal hematoma: Diagnosis and treatment. Two case reports. Ann Urol. 2002; 36:29.

4. Koo V, Duggan B, Lennon G. Spontaneous rupture of kidney with peri-renal haematoma: A conservative approach. Ulster Med J. 2004; 73:53-6.

5. Srinivasan V, Turner AG, Blackford HN. Massive intraperitoneal hemorrhage associated with renal pathology. J Urol. 1994; 151:980-1.

6. Mc Dougal WS, Kursh ED, Persky L. Spontaneous rupture of the kidney with perirenal hematoma. J Urol. 1975; 114:181-184.

7. Zhang JQ, Fieling JR, Zou KH. Etiology of spontaneous perirenal haemorrhage: a meta-analysis. J Urol. 2002; 167:1593-1596.

8. Sunga KL, Bellolio MF, Gilmore RM, Cabrera D. Spontaneous retroperitoneal hematoma: etiology, characteristics, managament and outcome. J Emer Med. 2012; 43:e157-e161.
9. Mabjeesh NJ, Matzkin H. Spontaneous subcapsular renal hematoma secondary to anticoagulant theraphy. J Urol 2001; 165:1201.

10. Zhu JP, Davidsen MB, Meyhoff HH. Aspirin, a silent risk factor in urology. Scand J Urol. Nephrol. 1995; 29:369-74

11. Mrug M, Mishra PV, Lusane HC, et al. Hemothorax and retroperitoneal hematoma after anticoagulation with enoxaparin. South Med. J 2002; 110:69-71.

12. Boumdin H, Ameur A, Lezrek M, et al. Spontaneous subcapsular hematoma of the kidney. Report of six cases. Ann Urol. 2002; 36:357.

13. Zissin R, Ellis M, Gayer C. The CT findings abdominal anticoagulant-related hematomas. Semin Ultrasound CT MR 2006; 27:117-25.

14) Balci NC, Sirvanci M, Tufek I, et al. Spontaneous retroperitoneal hemorrhage secondary to subcapsular renal hematoma: MRI findings. Magn Reson Imaging. 2001; 19:1145.

15. Morgentaler A, Belville JS, Tumeh SS, et al. Rational approach to evalution and management of spontaneous perirenal hemorrhage. Surg Gynecol Obstet. 1990; 170:121-5

16. Bosniak MA. Spontaneous subcapsular and perirenal hematomas. Radiology. 1989; 172:601-2.

\section{Correspondence}

Abdulmuttalip Simsek, MD (Corresponding Author)

simsek76@yahoo.com

Faruk Ozgor, MD

Mehmet Fatih Akbulut, MD

Onur Kucuktopcu, MD

Omer Sarilar, MD

Ahmet Yalcin Berberoglu, MD

Zafer Gokhan Gurbuz, MD

Haseki Training and Research Hospital, Department of Urology,

Millet Cad. No: 11, 34000 Fatih, Istanbul, Turkey

Bahar Yuksel, MD

Ercan Bastu, MD

Istanbul University, School of Medicine, Department of Obstetrics and Gynecology, Istanbul, Turkey 